ACCEPTED MANUSCRIPT

\title{
Deep-ultraviolet to mid-infrared polarizers by Al nanowire metamaterials
}

To cite this article before publication: Junxi Zhang et al 2019 J. Phys. D: Appl. Phys. in press https://doi.org/10.1088/1361-6463/ab28c9

\section{Manuscript version: Accepted Manuscript}

Accepted Manuscript is "the version of the article accepted for publication including all changes made as a result of the peer review process, and which may also include the addition to the article by IOP Publishing of a header, an article ID, a cover sheet and/or an 'Accepted Manuscript' watermark, but excluding any other editing, typesetting or other changes made by IOP Publishing and/or its licensors"

This Accepted Manuscript is @ 2019 IOP Publishing Ltd.

During the embargo period (the 12 month period from the publication of the Version of Record of this article), the Accepted Manuscript is fully protected by copyright and cannot be reused or reposted elsewhere.

As the Version of Record of this article is going to be / has been published on a subscription basis, this Accepted Manuscript is available for reuse under a CC BY-NC-ND 3.0 licence after the 12 month embargo period.

After the embargo period, everyone is permitted to use copy and redistribute this article for non-commercial purposes only, provided that they adhere to all the terms of the licence https://creativecommons.org/licences/by-nc-nd/3.0

Although reasonable endeavours have been taken to obtain all necessary permissions from third parties to include their copyrighted content within this article, their full citation and copyright line may not be present in this Accepted Manuscript version. Before using any content from this article, please refer to the Version of Record on IOPscience once published for full citation and copyright details, as permissions will likely be required. All third party content is fully copyright protected, unless specifically stated otherwise in the figure caption in the Version of Record.

View the article online for updates and enhancements. 


\title{
Deep-ultraviolet to mid-infrared polarizers by Al nanowire metamaterials
}

\author{
Junxi Zhang ${ }^{1,2}$, Kang Xie ${ }^{1}$, Yongqing Wei ${ }^{1}$, Pei Wang ${ }^{3}$, Zhijia Hu ${ }^{1,2,4}$, Haoyu Wang ${ }^{1}$, \\ Fei Wang ${ }^{1}$, Taiming Liang ${ }^{1}$, Sonia Ghafoor ${ }^{3}$, Haiming Jiang ${ }^{1}$, Lide Zhang $^{5}$ and Lin \\ Zhang $^{2}$
}

${ }^{1}$ Department of Opto-electronic Information Science and Engineering, School of Instrument Science and Opto-electronics Engineering, National Engineering Lab of Special Display Technology, Key Lab of Advanced Display Technology, Hefei University of Technology, Hefei 230009, P. R. China

${ }^{2}$ Aston Institute of Photonic Technologies, School of Engineering \& Applied Science, Aston University, Birmingham B4 7ET, UK

${ }^{3}$ Department of Optics and Optical Engineering, Anhui Key Laboratory of Optoelectronic Science and Technology, University of Science and Technology of China, Hefei 230026, P. R. China

${ }^{4}$ State Key Laboratory of Environment-Friendly Energy Materials, Southwest University of Science and Technology, Mianyang, Sichuan 621000, P. R. China

${ }^{5}$ Key Laboratory of Materials Physics and Anhui Key Laboratory of Nanomaterials and Nanostructures, Institute of Solid State Physics, Chinese Academy of Sciences, Hefei 230031, China

${ }^{5}$ Department of Optics and Optical Engineering, University of Science and Technology of China, Hefei 230026, P. R. China

E-mail: junxi.zhang@hfut.edu.cn, kangxie@hfut.edu.cn

Received xxxxxx

Accepted for publication $\mathrm{xxxxxx}$

Published xxxxxx

\begin{abstract}
Polarizers are one of the most fundamental elements used in optical devices. Compared with conventional prism polarizers, wire grid polarizers have potential for the miniaturization and integration of the optical devices. There are growing demands for design of the wire grid polarizers operating in a very broad spectral range from deep-ultraviolet to mid-infrared wavelengths. However, construction of the ultrabroadband polarizers based on the same structures and materials is very challenging. Here, ultrabroadband optical polarizers working in the deep-ultraviolet to mid-infrared spectral region, based on Al nanowire metamaterials with hexagonally packed nanowire arrays, are presented. To synchronously obtain high extinction ratios and low insertion losses, a $q$ factor is found, when the $q$ factor is fixed, the extinction ratio increases and the insertion loss simultaneously decreases with decreasing nanowire diameter. Moreover, the cut-off wavelength of the polarizers remarkably shifts to much shorter wavelength when the refractive index of the surrounding medium decreases. Consequently, the polarizers demonstrate high performance operating down to the deepultraviolet spectral range based on an optimal design of the Al nanowire metamaterials embedded in air by selecting a smaller diameter (e.g., $15 \mathrm{~nm}$ ) and a suitable $q$ factor (e.g., 2
\end{abstract} or 2.5).

Keywords: deep ultraviolet to mid-infrared spectral region, ultrabroadband polarizer, Al nanowire metamaterial, $q$ factor, cutoff wavelength 


\section{Introduction}

Polarizers that enable control the polarization state of light, are one of the most fundamental elements used in optical devices, such as switches, isolators, filters and modulators. Compared with the traditional prism polarizers, wire grid polarizers have potential for miniaturization and integration of the optical devices, which have been widely applied to many fields, including liquid-crystal display [1-5], imaging [6-8], beam splitters [5,9] and Bio-detection [10]. The wire grid polarizers in the infrared [11-17] and terahertz [18-22] spectral range have been reported widely. Recently, the polarizing elements working at shorter wavelengths have received growing attention. Visible wire grid polarizers can be constructed by metal [5,23,24], semiconductor [25], dielectric [26] wire grids. For example, a visible wire grid polarizer has been fabricated by using a method with a combination of nanoimprint lithography and angled metal evaporation [5]. Recently, a wire grid polarizer composed of solution-processed $\mathrm{Al}$ nanogratings with a line width of 60 $\mathrm{nm}$ and a height of $150 \mathrm{~nm}$ exhibits an average extinction ratio of 104 and transmittance of $52.3 \%$ in the visible spectral range [24]. Furthermore, a highly flexible and stretchable wire grid polarizer has been presented by printing bottom-up grown $\mathrm{Ge}$ or $\mathrm{Ge} / \mathrm{Si}$ core/shell nanowires on device substrates, the maximum contrast ratio of 104 between transverse electric and transverse magnetic fields and above $99 \%$ of light blocking efficiency across the visible spectral range can be achieved [25]. Based on a photonic structure consisting of a pair of subwavelength dielectric nanowires with anisotropic permittivity, a broadband and highly efficient optical polarizer working in the visible spectral range has been designed theoretically [26]. On the other hand, the wire grid polarizers working at the deep ultraviolet wavelengths have been mainly designed by semiconductor materials [27-29]. For instance, a titanium dioxide wire grid polarizer based on the semiconductor absorption from interband transition processes demonstrates a superior polarizing performance with an unprecedented extinction ratio of 384 and a transmittance of $10 \%$ at the deep ultraviolet wavelengths [28]. In addition, a silicon wire grid polarizer with a period of $140 \mathrm{~nm}$ exhibits an extinction ratio of $90(19.5 \mathrm{~dB})$ and a transmission of $42 \%$ at a wavelength of $365 \mathrm{~nm}$, the spectral bandwidth of the polarizer is about $100 \mathrm{~nm}[29]$.

As demonstrated above, the wire grid polarizers for the different spectral regions can be constructed by selecting different structures and materials, however, the working spectral region is narrow for the polarizing elements designed by one sort of configuration. There are growing demands for design of the wire grid polarizers covering very broad spectral range from the deep-ultraviolet (DUV) to midinfrared (MIR) wavelengths. Considering the ultrabroad spectral range, construction of the wire grid polarizers based on the same structures and materials is very challenging. In this paper, we propose ultrabroadband optical polarizers based on Al nanowire metamaterials with hexagonally packed nanowire arrays. The optical polarizers present very strong extinction characteristics due to the periodical multilayer configuration of wire grids, when compared with those composed of single layer structure of wire grids reported previously. Nevertheless, the insertion loss is not low due to the multilayer structure of the Al wire grids. So, how to decrease the insertion loss when the extinction ratio is very high, is of significance. In this case, we find a key parameter $q$ factor to synchronously obtain high extinction ratios and low insertion losses in the Al nanowire metamaterials, Moreover, the optical polarizers exhibit highly efficient performance in the whole spectral region of the deep-ultraviolet to mid-infrared wavelengths based on an optimal design of the Al nanowire metamaterials.

\section{Results and discussion}

\subsection{Calculation of optical losses of the Al nanowire metamaterials}

The Al nanowire metamaterial depicted here is a hexagonally packed nanowire array (Figure 1), which consists of an ordered arrangement of Al nanowires embedded in a medium (e.g., alumina or air), the nanowires with a diameter of $2 a$ and a spacing of $b$ are parallel to each other. Consider a light wave impinges on a lateral surface of the metallic $\mathrm{Al}$ nanowire metamaterial at normal incidence (Figure 1). The wave numbers $k_{1}$ and $k_{2}$ can be expressed as $k_{1}=2 \pi n_{1} / \lambda$ and $k_{2}=2 \pi n_{2} / \lambda$, here $\lambda$ is the wavelength of the incident wave in vacuum. $n_{1}$ represents the refractive index of the medium, for the alumina as the medium, $n_{1}$ gradually decreases with the wavelength in the deep ultraviolet to midinfrared spectral range [30], for the air as the medium, $n_{1}=1$. The optical constant $n_{2}$ (complex refractive index) of the metal can be written as $n_{2}=n_{s}-i k$ [31], where $n_{s}$ and $k$ are the refractive index and extinction coefficient of $\mathrm{Al}$ [32], respectively. TE and TM represent TE and TM polarized waves, their electric fields of the two incident waves, corresponding to light polarized parallel (s-polarization) and perpendicular (p-polarization) to the nanowire axis. The extinction ratio (ER) and insertion loss (IL)) of the Al nanowire metamaterial are given by

$$
\mathrm{ER}=\left(\alpha_{s}-\alpha_{p}\right) d=-\left(\log T_{s}-\log T_{p}\right)
$$

and

$$
\mathrm{IL}=\alpha_{p} d=-\log T_{p}
$$


Where $\alpha_{s}$ and $\alpha_{p}$ are the optical losses per thickness of the metamaterial, $d$ is the thickness of the metamaterial along the incident direction. The transmittance $T_{s}$ and $T_{p}$ through the nanowire metamaterial for the TE and TM polarized waves can be written as

$$
T_{s}=T_{0 s}{ }^{m} ; T_{p}=T_{0 p}{ }^{m}
$$

$m$ denotes the row number of the nanowires, and $m=\frac{2 d}{\sqrt{3} b}+1$, where $d$ is the thickness of the metamaterial along the incident direction. $T_{0 s}$ and $T_{0 p}$ represent the transmittance through a row of the nanowires, and we have

$$
T_{0 s}=\left|t_{s}\right|^{2} ; T_{0 p}=\left|t_{p}\right|^{2}
$$

Where $t_{s}$ and $t_{p}$ are the transmission coefficient of a row of the nanowires for TE and TM polarized waves, respectively. In terms of Equations (1) to (4), ER and IL can be expressed as

$$
\mathrm{ER}=-2 m\left(\log \left|t_{s}\right|-\log \left|t_{p}\right|\right)
$$

and

$$
\mathrm{IL}=-2 m \log \left|t_{p}\right|
$$

Here the transmission coefficient takes the form [33]

$$
t_{i}=1-\left[1-\frac{i b \eta_{1 i}}{\pi k_{1} a^{2}}\right]^{-1}-\left[1+\frac{i k_{1} b\left(\eta_{0 i}-\log 2\right)}{\pi}\right]^{-1}
$$

where $i=s, p, \eta_{0 i}$ and $\eta_{1 i}$ are two parameters dependent on the polarization direction. Furthermore, the quantities can be obtained by using the boundary conditions, for TE polarization the parameters are expressed as

$$
\begin{gathered}
\eta_{0 s}=\frac{J_{0}\left(k_{2} a\right)-\left(k_{2} a\right) J_{0}{ }^{\prime}\left(k_{2} a\right) \log (\pi a / b)}{\left(k_{1} a\right)^{2} J_{0}\left(k_{2} a\right) / 2+\left(k_{2} a\right) J_{0}^{\prime}\left(k_{2} a\right)} \\
\eta_{1 s}=\frac{J_{1}\left(k_{2} a\right)+\left(k_{2} a\right) J_{1}^{\prime}\left(k_{2} a\right)}{J_{1}\left(k_{2} a\right)-\left(k_{2} a\right) J_{1}^{\prime}\left(k_{2} a\right)}
\end{gathered}
$$

For TM polarization, one has

$$
\begin{gathered}
\eta_{0 p}=\frac{\left(k_{2} a\right) J_{0}\left(k_{2} a\right) /\left(k_{1} a\right)^{2}-J_{0}{ }^{\prime}\left(k_{2} a\right) \log (\pi a / b)}{\left(k_{2} a\right) J_{0}\left(k_{2} a\right) / 2+J_{0}{ }^{\prime}\left(k_{2} a\right)} \\
\eta_{1 p}=\frac{\left(k_{2} a\right) J_{1}\left(k_{2} a\right)+\left(k_{1} a\right)^{2} J_{1}^{\prime}\left(k_{2} a\right)}{\left(k_{2} a\right) J_{1}\left(k_{2} a\right)-\left(k_{1} a\right)^{2} J_{1}^{\prime}\left(k_{2} a\right)}
\end{gathered}
$$

where $J_{0}, J_{1}$ are Bessel functions of 0 and 1 orders, and $J_{0}{ }^{\prime}$, $J_{1}$ ' correspond to their derivatives, respectively.

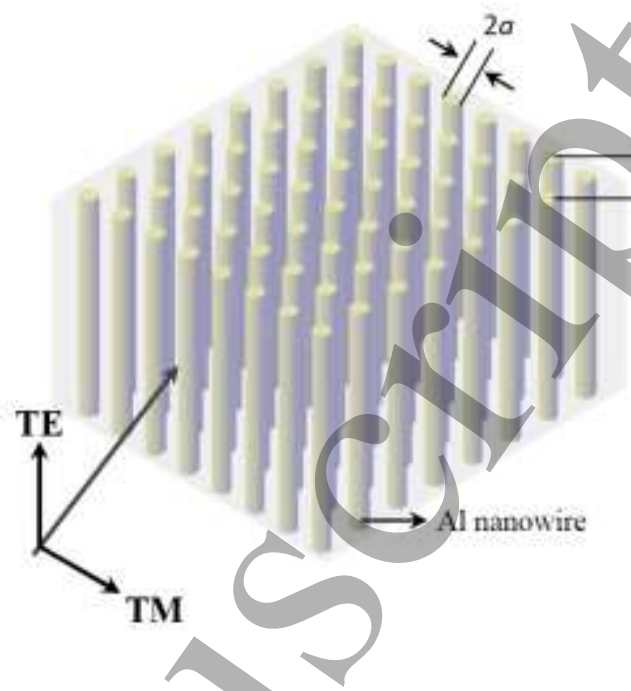

Figure 1. Schematic illustration of an $\mathrm{Al}$ nanowire metamaterial consisted of a hexagonally packed nanowire array, the nanowires are $2 a$ in diameter and $b$ in spacing. TE and TM polarized waves illuminate a lateral surface of the metamaterial at normal incidence.

2.2 Diameter and spacing dependence of the polarization properties of the Al nanowire metamaterials

Figure 2 shows the optical losses with the nanowire diameter of the $\mathrm{Al}$ nanowire arrays embedded in a dielectric (e.g., alumina) when the nanowire spacing is $100 \mathrm{~nm}$ for a constant thickness of the metamaterials (e.g., $10 \mu \mathrm{m}$ ).

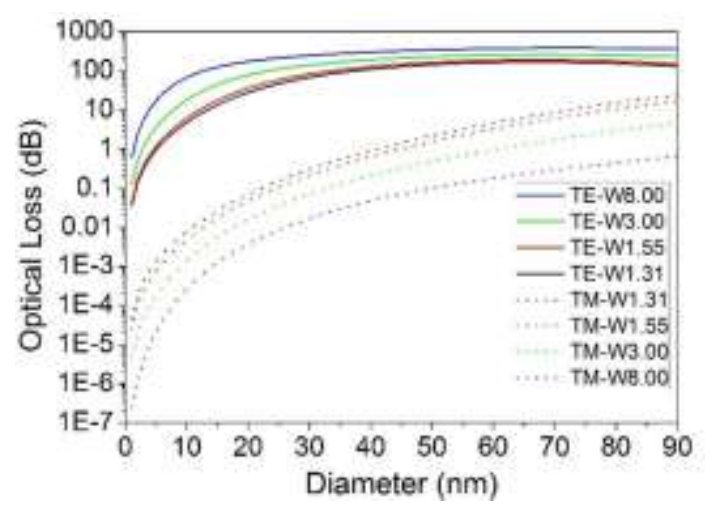

Figure 2. Optical losses with the nanowire diameter of the $\mathrm{Al}$ nanowire metamaterials for a constant spacing of $100 \mathrm{~nm}$ at different wavelengths, where the black, red, green and blue solid curves correspond the extinction ratios at the wavelengths of 1.31 , $1.55,3.00$ and $8.00 \mu \mathrm{m}$, respectively, while the black, red, green and blue dot curves represent the insertion losses at 1.31, 1.55, 3.00 and $8.00 \mu \mathrm{m}$. TE and TM denote the TE and TM polarized waves, respectively. 
Here some typical wavelengths in the near infrared spectral region, such as $1.31,1.55,3.00$ and $8.00 \mu \mathrm{m}$, are selected, where $1.31 \mu \mathrm{m}$ and $1.55 \mu \mathrm{m}$ are two main wavelengths for optical fiber communications, and 3-5 $\mu \mathrm{m}$ and 8-12 $\mu \mathrm{m}$ are thermo imaging wavebands and thermo infrared wavelengths for atmospheric window. The extinction ratio increases but the insertion loss decreases as the wavelength increases from $1.31 \mu \mathrm{m}$ to $8.00 \mu \mathrm{m}$, indicating the $\mathrm{Al}$ nanowire metamaterials present much better polarization characteristics at longer wavelengths. Moreover, both the extinction ratio and insertion loss basically increase with increasing the nanowire diameter, note that the insertion loss increases sharply as the diameter increases, while the extinction ratio increase slowly when the diameter is larger than $50 \mathrm{~nm}$. Since the high extinction ratio originates from the Fresnel reflection and absorption from the Al nanowire arrays with the periodical multilayer configuration, and the insertion loss results from the Raleigh scattering from the nanowire arrays [33,34], when the nanowire diameter increases for the constant spacing of $100 \mathrm{~nm}$, there is an increasing amount of metal to reflect and absorb the incident light for TE polarization, so the extinction ratio increases, while, the metal with an increase in fraction contributes to the Rayleigh scattering for the incident TM polarized light, as a result, the insertion loss also enhances.

The optical losses with the nanowire spacing when the diameter of the $\mathrm{Al}$ nanowire metamaterials is fixed to $40 \mathrm{~nm}$, are illustrated in Figure 3. It is observed that the extinction ratio increases while the insertion loss decreases when the wavelength increases from $1.31 \mu \mathrm{m}$ to $8.00 \mu \mathrm{m}$, which also demonstrates the Al nanowire metamaterials have much better polarization performance at longer wavelengths.

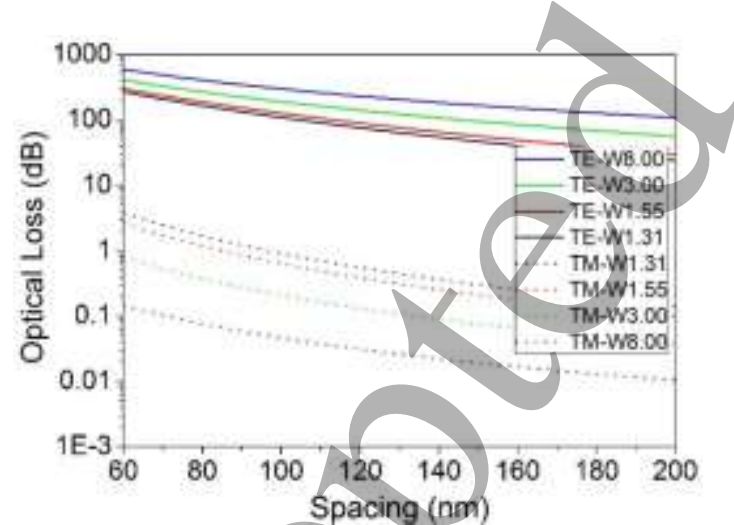

Figure 3. Optical losses with the nanowire spacing of the $\mathrm{Al}$ nanowire metamaterials for a constant diameter of $40 \mathrm{~nm}$ at different wavelengths, where the black, red, green and blue solid curves correspond the extinction ratios at the wavelengths of 1.31, $1.55,3.00$ and $8.00 \mu \mathrm{m}$, respectively, while the black, red, green and blue dot curves represent the insertion losses at 1.31, 1.55, 3.00 and $8.00 \mu \mathrm{m}$ wavelengths. TE and TM represent the TE and TM polarized waves, respectively.
Furthermore, both the extinction ratio and insertion loss reduce when the nanowire spacing rises. This is because that the number of the nanowire reduces with increasing the nanowire spacing for the same irradiation area of the incident light, consequently, there is a decreasing amount of metal to reflect and absorb the incident TE polarized light, as well as a reduction of the $\mathrm{Al}$ nanowires take part in the Rayleigh scattering for the incident TM polarized light.

\subsection{Mechanisms on modes and resonances of the Al nanowire metamaterials for different polarized waves at normal incidence.}

Furthermore, the mechanisms on the mode and the resonance characteristics of the $\mathrm{Al}$ nanowire metamaterials can be investigated in the near field regions of the metamaterials. Figure 4 illustrates the electric field distributions of the Al nanowire metamaterials by a finitedifference time-domain (FDTD) method with Lumerical package for $\mathrm{s}$ and $\mathrm{p}$ polarized waves at the normal incidence, where the simulation domain covers an $\mathrm{Al}$ nanowire with an arrangement of hexegonal lattice under the perfectly matched layer (PML) boundary conditions in the $\mathrm{z}$ direction and the periodic boundary conditions in the $\mathrm{x}$ and $\mathrm{y}$ directions. The electric field profile $\mathrm{E}(\mathrm{x}, \mathrm{z})$ of the $\mathrm{Al}$ nanowire metamaterial exhibits a resonance behavior at the outer interface along the
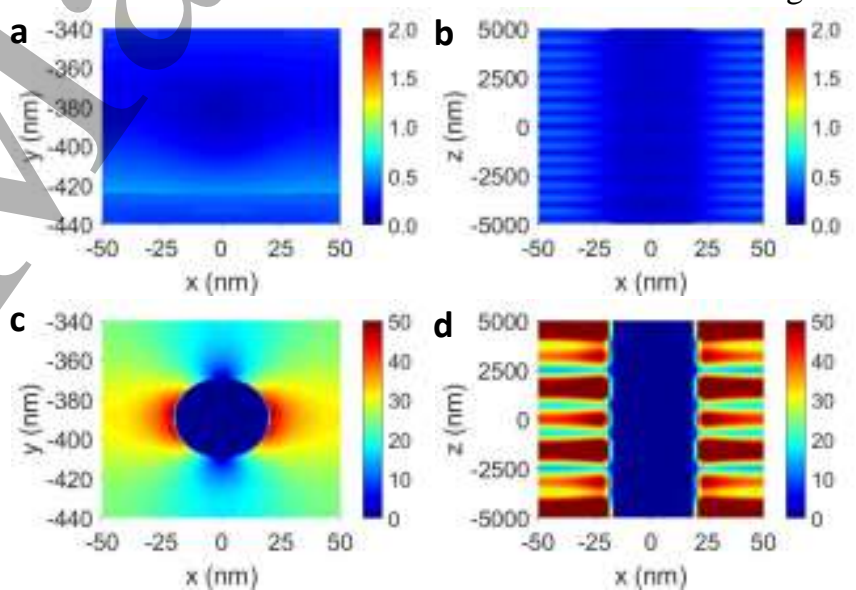

Figure 4. The electric field distributions of the $\mathrm{Al}$ nanowire metamaterials based on FDTD simulations, where, the nanowire array has a diameter of $20 \mathrm{~nm}$, a spacing of $100 \mathrm{~nm}$ and a length of $10 \mu \mathrm{m}$, the perfectly matched layer (PML) boundary conditions in the $\mathrm{z}$ direction and the periodic boundary conditions in the $\mathrm{x}$ and $\mathrm{y}$ directions are selected in the simulation domain. (a) and (b) represent the electric field $(|\mathbf{E}|)$ profiles of $\mathrm{E}(\mathrm{x}, \mathrm{y})$ and $\mathrm{E}(\mathrm{x}, \mathrm{z})$ when a s-polarized light at $1550 \mathrm{~nm}$ wavelength illuminates the $\mathrm{Al}$ nanowire array at normal incidence along y direction, where a $2 \mathrm{D} \mathrm{z}$ normal monitor $(\mathrm{z}=0)$ and a $2 \mathrm{D} y$-normal monitor $(\mathrm{y}=0)$ are used to detect the electric field distributions of the nanowire metamaterial in $x-y$ plane and $z-x$ plane, respectively. (c) and (d) correspond the electric field $(|\mathbf{E}|)$ profiles of $\mathrm{E}(\mathrm{x}, \mathrm{y})$ and $\mathrm{E}(\mathrm{x}, \mathrm{z})$ when a p-polarized light at $1550 \mathrm{~nm}$ wavelength illuminates the $\mathrm{Al}$ nanowire array at normal incidence along y direction. 
long axis of the nanowires and performs a standing wave mode for the s-polarized light with the $\mathrm{z}$ polarization direction (Figure 4b). Moreover, for the p-polarized light with the $\mathrm{x}$ polarization direction, very strong electric fields localized along the $\mathrm{x}$ direction can be observed (Figure $4 \mathrm{c}$ and $4 \mathrm{~d}$ ), which results from the electric field coupling between the adjacent nanowires. On the other hand, the electric fields along the $\mathrm{x}$ direction for a single $\mathrm{Al}$ nanowire is much lower than those for the $\mathrm{Al}$ nanowire metamaterial (Figure $5 \mathrm{c}$ and $5 \mathrm{~d}$ ), indicating that the strong electric fields are attributed to the coupling between the adjacent nanowires for the p-polarized light along the $\mathrm{x}$ polarization direction (Figure 4d). It is noted that the electric field along the $x$ direction for the single $\mathrm{Al}$ nanowire cannot present a coupling mode (Figure 5d).

\subsection{A key parameter: $q$ factor to determine the polarization performance of the Al nanowire metamaterials}

As demonstrated above, both the extinction ratio and insertion loss simultaneously rise by increasing the diameter or decreasing the spacing of the nanowires, also, the extinction ratio and insertion loss simultaneously reduce by decreasing the nanowire diameter or increasing the nanowire spacing. Consider design of the optical polarizers with high performance, what one aims for is how to achieve high
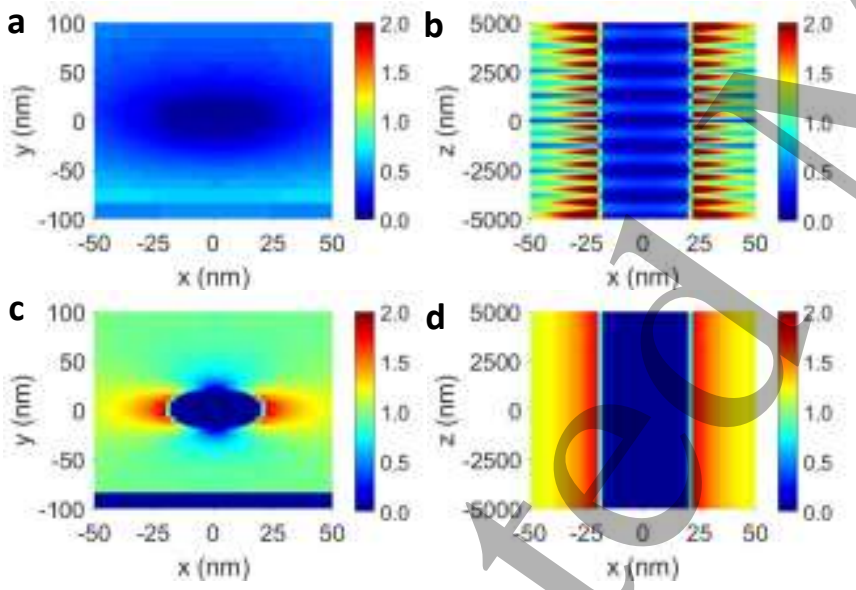

Figure 5. The electric field distributions of an $\mathrm{Al}$ nanowire based on FDTD simulations. Where the nanowire has a diameter of $20 \mathrm{~nm}$ and a length of $10 \mu \mathrm{m}$, the PML boundary conditions in the $\mathrm{x}, \mathrm{y}$ and $\mathrm{z}$ directions are used in the simulation domain. (a) and (b) represent the electric field $(|\boldsymbol{E}|)$ profiles of $\mathrm{E}(\mathrm{x}, \mathrm{y})$ and $\mathrm{E}(\mathrm{x}, \mathrm{z})$ when a spolarized light at $1550 \mathrm{~nm}$ wavelength illuminates the $\mathrm{Al}$ nanowire at normal incidence along y direction, where a $2 \mathrm{D}$ z-normal monitor $(\mathrm{z}=0)$ and a $2 \mathrm{D} y$-normal monitor $(\mathrm{y}=0)$ are used to detect the electric field distributions of the nanowire in $x-y$ plane and $z-x$ plane, respectively. (c) and (d) correspond the electric field $(|\boldsymbol{E}|)$ profiles of $\mathrm{E}(\mathrm{x}, \mathrm{y})$ and $\mathrm{E}(\mathrm{x}, \mathrm{z})$ when a p-polarized light at $1550 \mathrm{~nm}$ wavelength illuminates the $\mathrm{Al}$ nanowire at normal incidence along $\mathrm{y}$ direction. extinction ratios and low insertion losses. Obviously, this aim cannot be achieved by changing the diameter or the spacing of the $\mathrm{Al}$ nanowire independently.

Note that the periodical multilayer $\mathrm{Al}$ nanowires results in a very high extinction ratio owing to their strong Fresnel reflection and absorption. However, the insertion loss is not low. So, how to decrease the insertion loss is challenging. To synchronously obtain high extinction ratios and low insertion losses in the Al nanowire metamaterials, we find a key parameter, which is named as $q$ factor, the $q$ factor is defined as $q=b / 2 a-1$, it represents the ratio of the gap between two adjacent nanowires to the diameter of the nanowires. Figure 6 displays the diameter dependence of optical loss spectra for different $q$ factors (e.g., 0, 1, 2, 3, 5 and 10). For every fixed $q$ factor, the extinction ratio increases, and the insertion loss decreases when the nanowire diameter reduces from $80 \mathrm{~nm}$ to $20 \mathrm{~nm}$.

This result illustrated above can be explained further. As the nanowire diameter decreases, the nanowire spacing also reduces for those fixed $q$ factors, which gives rise to the increase of the number of nanowires $n\left(n \propto 1 / b^{2}\right)$. It is clear the Fresnel reflection and absorption from the $\mathrm{Al}$ nanowires reduce as the nanowire diameter decreases, but the reflection and absorption rise when the nanowire spacing decrease because of the increase of the nanowire number. In this case, the Fresnel reflection and absorption increase with decreasing the nanowire diameter, because they result from the contribution from both the decrease of the nanowire diameter/ and the increase of the nanowire number, nevertheless, the contribution from the nanowire number may be larger than that from the nanowire diameter, as a result, the extinction ratio increases when the nanowire diameter decreases. On the other hand, the insertion loss stems from the Raleigh scattering from the nanowires, which exhibits a remarkable decline when the nanowire diameter reduces because the Rayleigh scattering loss is proportional to $a^{2} / \lambda^{4}$ for the same wavelengths [33].

Although higher extinction ratios and lower insertion losses can be achieved simultaneously by fixing the $q$ factor and selecting a smaller nanowire diameter (e.g., $20 \mathrm{~nm}$ ), the $q$ factor has a dominant influence on the polarization performance of the Al nanowire metamaterials. Both the extinction ratio and insertion loss remarkably reduce with increasing the $q$ factor from $0,1,2,3,5$ to 10 for the same diameters and the same wavelengths (Figure $6 \mathrm{a}, \mathrm{b}, \mathrm{c}, \mathrm{d}$, e and $\mathrm{f}$ ). The $q$ factor dependence of the optical losses at different wavelengths can also be illustrated clearly when selecting a constant diameter of $20 \mathrm{~nm}$ in Figure 7. Based on Figure 7, the optical losses can be further analyzed quantitatively. For the same diameter of $20 \mathrm{~nm}$ and the same wavelength of $1.55 \mu \mathrm{m}$, the extinction ratios are $819.33 \mathrm{~dB}$, $248.45 \mathrm{~dB}, 111.19 \mathrm{~dB}, 59.55 \mathrm{~dB}, 23.04 \mathrm{~dB}$ and $5.09 \mathrm{~dB}$ as 
a

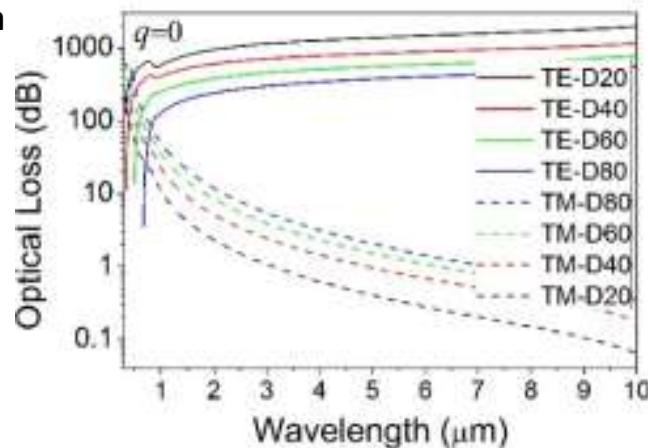

C

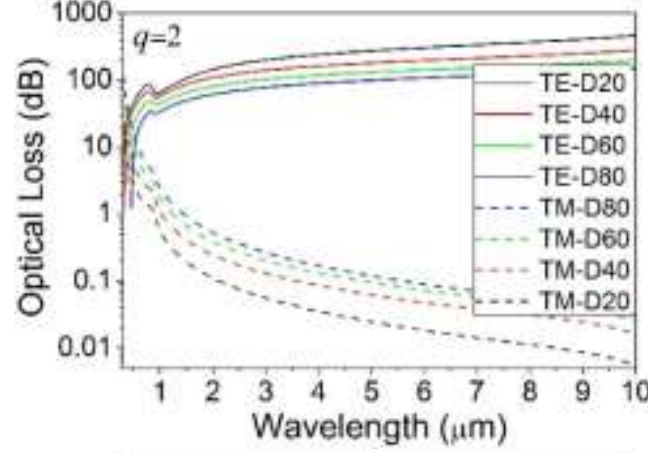

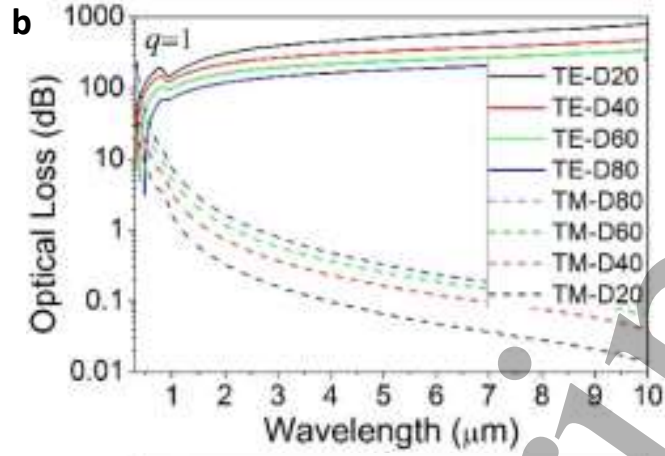
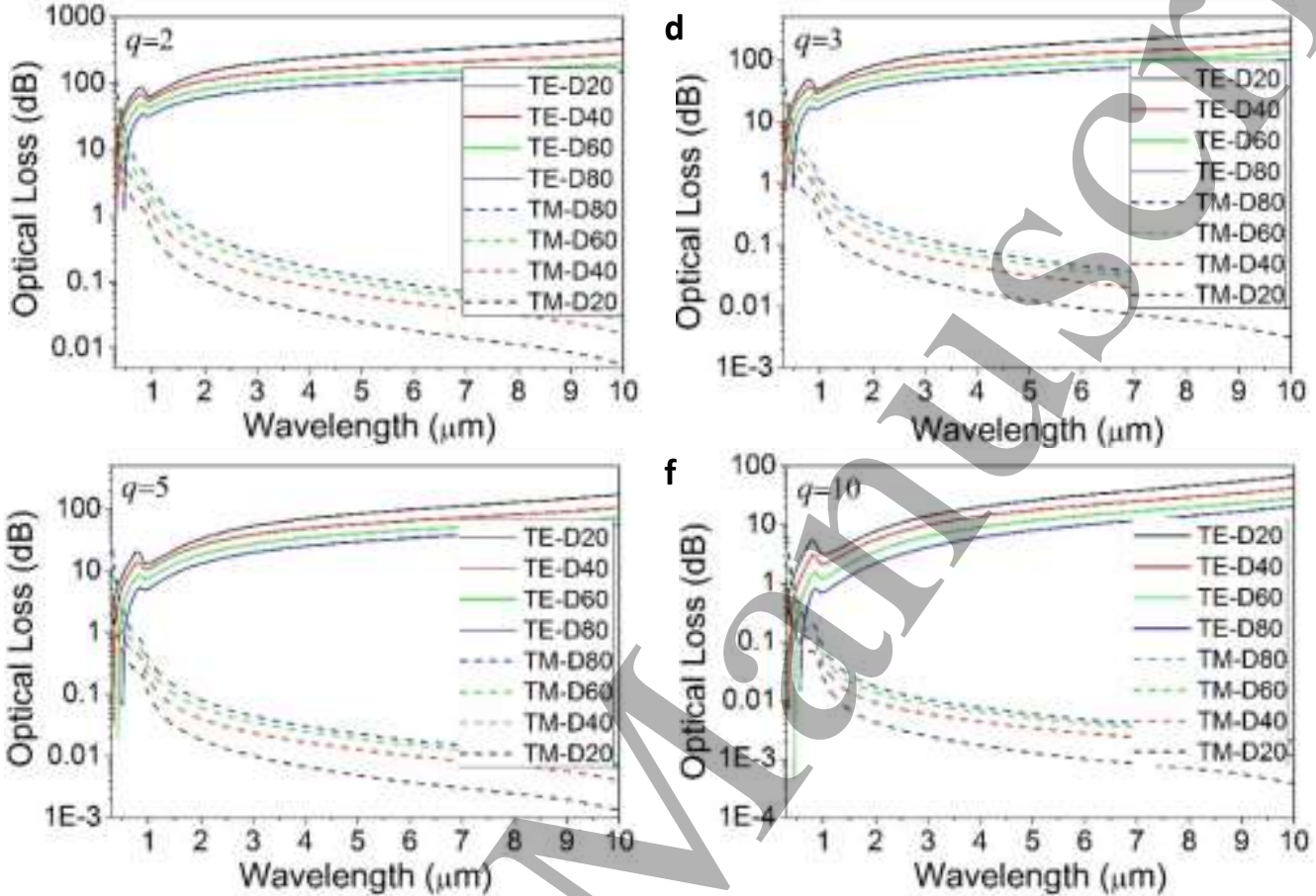

Figure 6. Optical loss spectra of the $\mathrm{Al}$ nanowire metamaterials with different diameters of the nanowires (e.g., $20 \mathrm{~nm}, 40 \mathrm{~nm}, 60 \mathrm{~nm}$ and $80 \mathrm{~nm}$ ) for the fixed $q$ factors. (a), (b), (c), (d), (e) and (f) correspond to the constant $q$ factors of 0, 1, 2, 3, 5 and 10, respectively. TE and TM denote the TE and TM polarized waves, respectively.

the $q$ factors are $0,1,2,3,5$ and 10 , respectively, while the insertion losses are $3.86 \mathrm{~dB}, 0.54 \mathrm{~dB}, 0.18 \mathrm{~dB}, 0.03 \mathrm{~dB}$ and $0.01 \mathrm{~dB}$ when the $q$ factors are $0,1,2,3,5$ and 10 , respectively (Table 1 ). In this case, as selecting $q=1,2$ or 3 , the optical polarizers exhibit excellent performances at 1.55 $\mu \mathrm{m}$, corresponding the extinction ratio of $248.45 \mathrm{~dB}$ and the insertion loss of $0.54 \mathrm{~dB}$ for $q=1$, the extinction ratio of $111.19 \mathrm{~dB}$ and the insertion loss of $0.18 \mathrm{~dB}$ for $q=2$, or the extinction ratio of $59.55 \mathrm{~dB}$ and the insertion loss of $0.03 \mathrm{~dB}$ for $q=2$. Therefore, one can design the optical polarizers with higher extinction ratios and lower insertion losses by selecting a constant $q$ factor and a smaller diameter of the nanowire (e.g., $20 \mathrm{~nm}$ ), particularly, the value of $q$ factor should be chosen reasonably based on the expected performance tolerance of the polarizers. For longer wavelengths (e.g., in the mid-infrared spectral region), the polarizer presents very high extinction ratios and very low

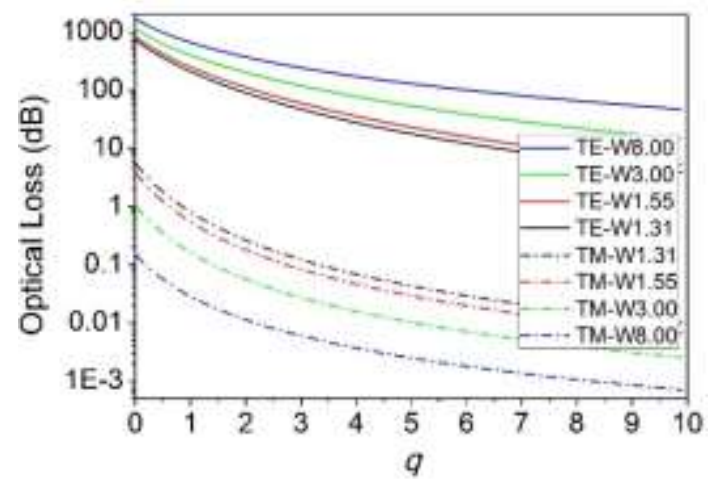

Figure 7. Optical losses of the $\mathrm{Al}$ nanowire metamaterials with the $q$ factor for a constant diameter of $20 \mathrm{~nm}$ at different wavelengths, where the black, red, green and blue solid curves correspond the extinction ratios at the wavelengths of $1.31,1.55,3.00$ and $8.00 \mu \mathrm{m}$, respectively, while the black, red, green and blue dash dot curves represent the insertion losses at the wavelengths of 1.31, 1.55, 3.00 and $8.00 \mu \mathrm{m}$. 
a

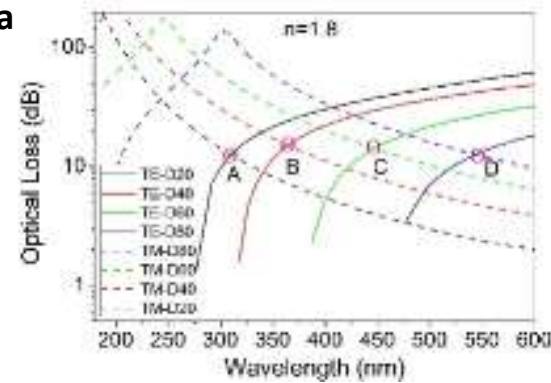

d

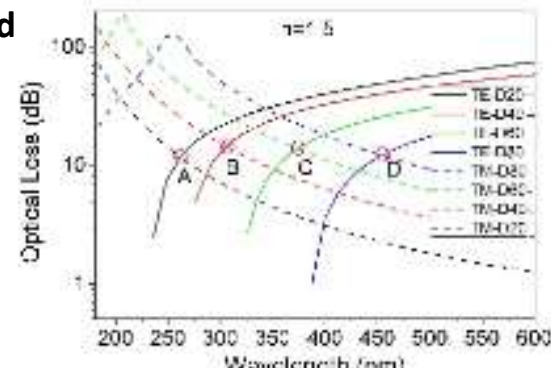
Wavclengith \{nm\}

g

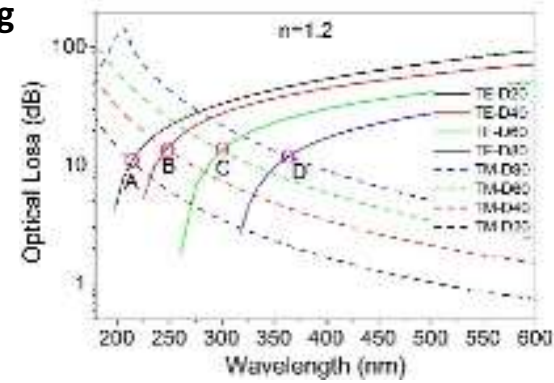

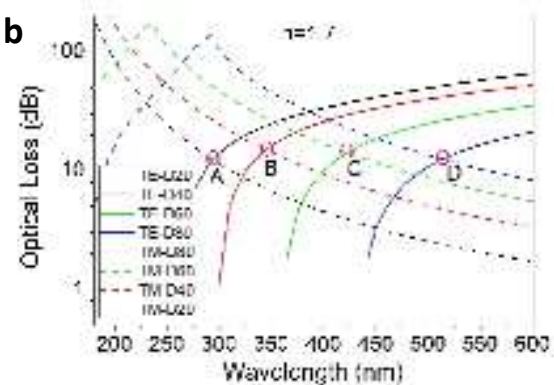
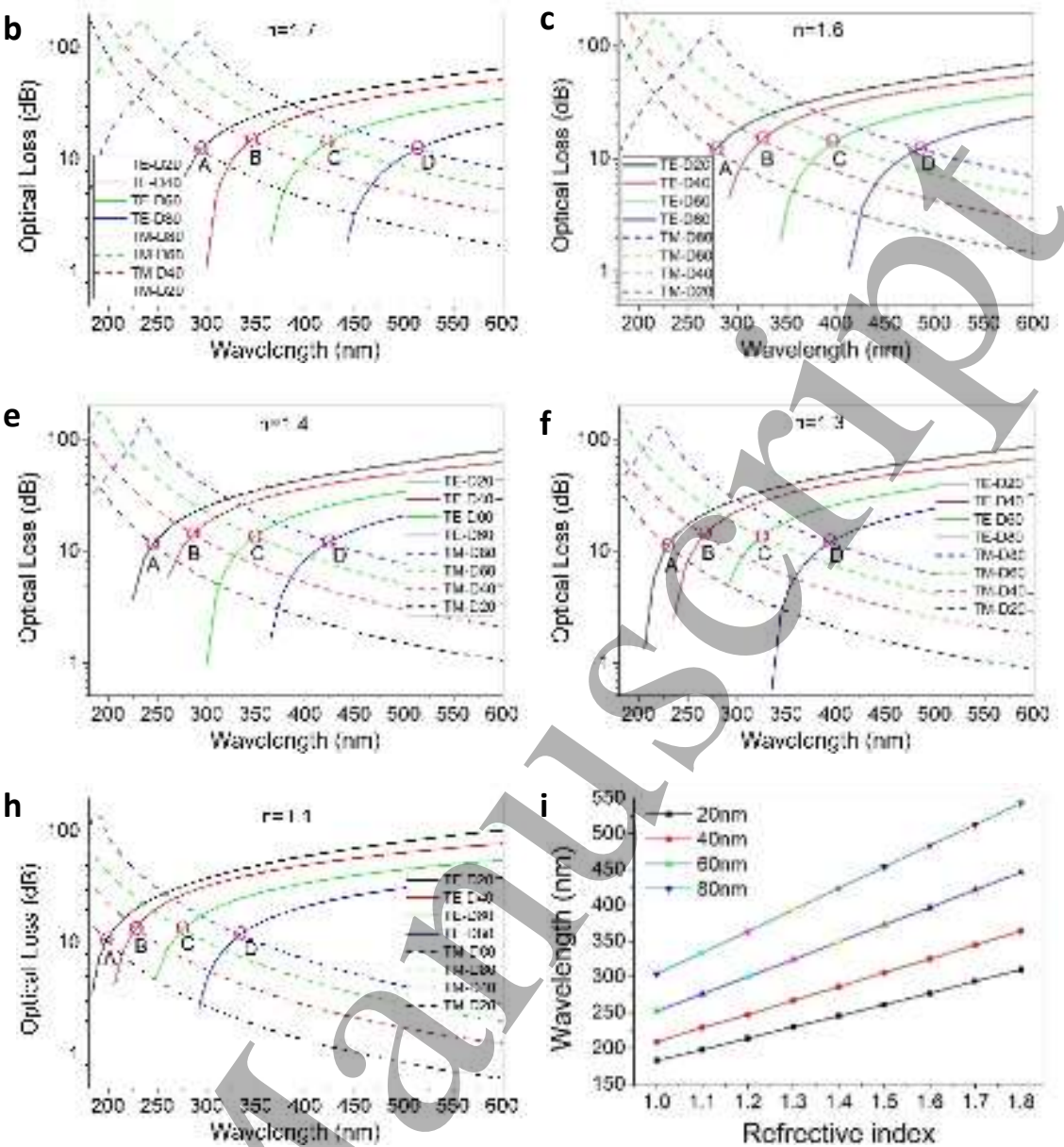

Figure 8. Optical loss spectra of the Al nanowire metamaterials embedded in dielectric materials with different refractive indexes for a fixed $q$ factor of 2 and the different diameters of the nanowires (e.g., $20 \mathrm{~nm}, 40 \mathrm{~nm}, 60 \mathrm{~nm}$ and $80 \mathrm{~nm}$ ). (a-h) correspond to the optical losses for the different refractive indexes of 1.8, 1.7, 1.6, 1.5, 1.4, 1.3, 1.2 and 1.1, respectively, where circles A, B, C and D denote the cutoff wavelengths. (i) represents the cut-off wavelengths with the refractive indexes for the different nanowire diameters of $20 \mathrm{~nm}, 40 \mathrm{~nm}, 60$ $\mathrm{nm}$ and $80 \mathrm{~nm}$, respectively.

insertion loss for almost any $q$ factors, but for shorter wavelengths (e.g., in the visible and ultraviolet spectral range), the $q$ should be a suitable value (e.g., 2), this case will be discussed further in the following section.

\subsection{Optimal design of the optical polarizers in the deep ultraviolet spectral region}

Note that the extinction ratio is smaller, and the insertion loss is larger for the ultraviolet spectral range when compared with that for the infrared spectral region. Moreover, the dielectric materials have a significant influence on the working spectral range of the polarizers. Consider the polarizers by the $\mathrm{Al}$ nanowire metamaterials with the nanowire diameter of $80 \mathrm{~nm}$, it is found that the cut-off wavelength remarkably decreases from $543 \mathrm{~nm}$ to $334 \mathrm{~nm}$
Table 1. Optical losses of the Al nanowire metamaterials embedded in alumina with the $q$ factor for the $20 \mathrm{~nm}$ diameter at the wavelength of $1.55 \mu \mathrm{m}$.

\begin{tabular}{ccc}
\hline$q$ & $\begin{array}{c}\text { Extinction ratio } \\
(\mathrm{dB})\end{array}$ & $\begin{array}{c}\text { Insertion loss } \\
(\mathrm{dB})\end{array}$ \\
\hline 0 & 819.33 & 3.86 \\
1 & 248.45 & 0.54 \\
2 & 111.19 & 0.18 \\
3 & 59.55 & 0.08 \\
5 & 23.04 & 0.03 \\
10 & 5.09 & 0.01 \\
\hline
\end{tabular}


along short wavelengths with decreasing the refractive index of the surrounding materials from 1.8 to 1.1 (Figure 8a-h). Moreover, the cut-off wavelength for the small nanowire diameter (e.g., $20 \mathrm{~nm}$ ) is shorter than that for the large nanowire diameter (e.g., $80 \mathrm{~nm}$ ), that is the cut-off wavelength reduces with decreasing the nanowire diameter from $80 \mathrm{~nm}$ to $20 \mathrm{~nm}$ (Figure $8 \mathrm{a}$ ). Nevertheless, the cut-off wavelength for the small nanowire diameter of $20 \mathrm{~nm}$ presents obvious blueshift from $310 \mathrm{~nm}$ to $198 \mathrm{~nm}$ when the refractive index decreases (Figure 8i). Therefore, the $\mathrm{Al}$ nanowire metamaterials embedded in the materials with lower refractive index exhibit obvious polarization performance at the deep-ultraviolet wavelengths when selecting a constant $q$ factor and a smaller nanowire diameter.

Furthermore, one can improve the polarization properties of the optical polarizers operating down to the deep ultraviolet region by an optimal design of the $q$ factor and the diameter of the $\mathrm{Al}$ nanowire metamaterials embedded in air. Figure 9 shows the optical loss spectra of the Al nanowire metamaterials embedded in air with the suitable $q$ factors (e.g., 2, 2.5, 3 and 3.5) and the smaller diameters (e.g., 15 $\mathrm{nm}, 20 \mathrm{~nm}$ and $25 \mathrm{~nm}$ ) in the ultraviolet and visible spectral ranges. It is observed that the $\mathrm{Al}$ nanowire metamaterials present almost the same extinction ratios but the dominantly different insertion losses in the ultraviolet wavelength range for the small diameters. The Al nanowire metamaterial of 15 $\mathrm{nm}$ in diameter exhibits much lower insertion loss at a fixed wavelength for the constant $q$ factors, indicating a higher polarization performance. Table 2 and 3 illustrate the extinction ratios and the insertion losses of the optical polarizers with the nanowire diameter of $15 \mathrm{~nm}$ at the typical deep-ultraviolet wavelengths, the $\mathrm{Al}$ nanowire metamaterials embedded in air at the wavelength of $207 \mathrm{~nm}$ exhibit an obvious polarization phenomenon, but the insertion losses are larger than that at other deep ultraviolet wavelengths for the same $q$ factors. Nevertheless, the nanowire metamaterials display high polarization performance at the wavelength of $248 \mathrm{~nm}$, for example, the extinction ratio and the insertion loss are $28.31 \mathrm{~dB}$ and $2.74 \mathrm{~dB}$ for the $q$ factor of 2 , respectively, the two optical losses correspond to $19.03 \mathrm{~dB}$ and $1.82 \mathrm{~dB}$ for the $q$ factor of 2.5. In addition, the $\mathrm{Al}$ nanowire metamaterials display very high performance as optical polarizers at the $350 \mathrm{~nm}$ wavelength, for instance, the extinction ratio and the insertion loss are $53.42 \mathrm{~dB}$ and 1.12 $\mathrm{dB}$ for the $q$ factor of 2 , respectively, they correspond to $36.68 \mathrm{~dB}$ and $0.74 \mathrm{~dB}$ for the $q$ factor of 2.5. As a result, the optical polarizers demonstrate high performance operating down to the deep-ultraviolet wavelength range based on the optimal design Al nanowire metamaterials embedded in air by selecting the smaller diameter (e.g., $15 \mathrm{~nm}$ ) and the suitable $q$ factor (e.g., 2 or 2.5).
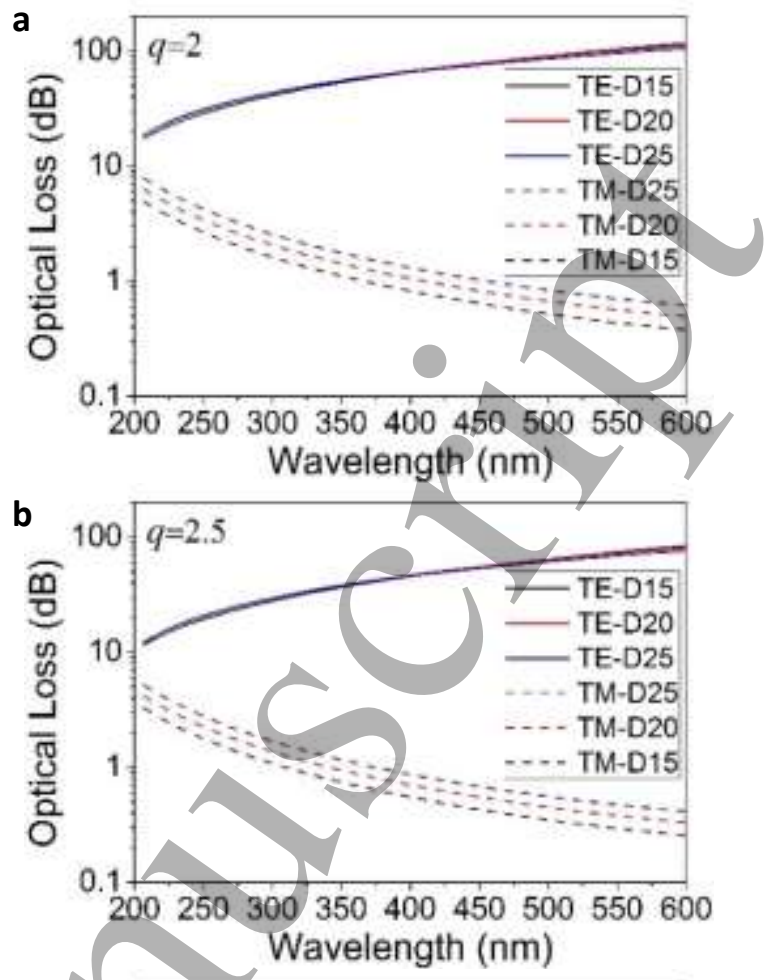

C
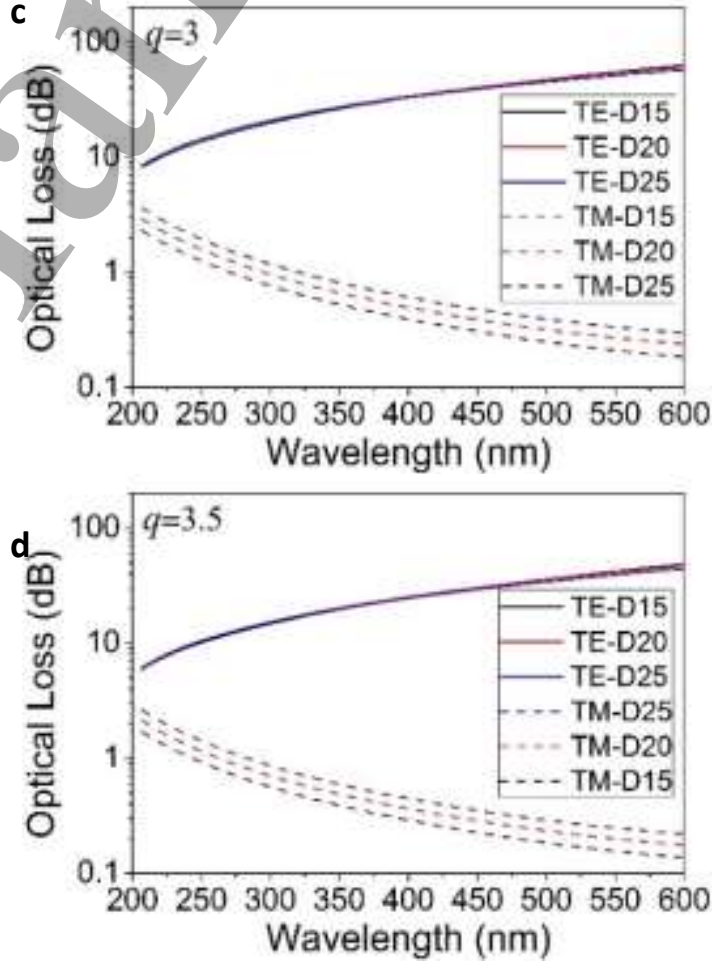

Figure 9. Optical loss spectra of the Al nanowire metamaterials embedded in air with different diameters (e.g., $15 \mathrm{~nm}, 20 \mathrm{~nm}$ and 25 $\mathrm{nm}$ ) for the fixed $q$ factors. (a), (b), (c) and (d) correspond to the constant $q$ factors of 2, 2.5, 3 and 3.5. 
Table 2. Extinction ratio (ER) of the Al nanowire metamaterials embedded in air with the fixed $q$ factors at the different wavelengths of 207, 248, 300 and $350 \mathrm{~nm}$.

\begin{tabular}{ccccc}
\hline$q$ & ER $(\mathrm{dB})$ & ER $(\mathrm{dB})$ & ER $(\mathrm{dB})$ & ER $(\mathrm{dB})$ \\
& $207 \mathrm{~nm}$ & $248 \mathrm{~nm}$ & $300 \mathrm{~nm}$ & $350 \mathrm{~nm}$ \\
\hline 2 & 17.43 & 28.31 & 41.06 & 53.42 \\
2.5 & 11.60 & 19.03 & 27.90 & 36.68 \\
3 & 8.15 & 13.47 & 19.91 & 26.38 \\
3.5 & 5.97 & 9.93 & 14.76 & 19.68 \\
\hline
\end{tabular}

Table 3. Insertion loss (IL) of the $\mathrm{Al}$ nanowire metamaterials embedded in air with the fixed $q$ factors at the different wavelengths of 207, 248, 300 and $350 \mathrm{~nm}$.

\begin{tabular}{ccccc}
\hline$q$ & IL $(\mathrm{dB})$ & $\mathrm{IL}(\mathrm{dB})$ & $\mathrm{IL}(\mathrm{dB})$ & $\mathrm{IL}(\mathrm{dB})$ \\
& $207 \mathrm{~nm}$ & $248 \mathrm{~nm}$ & $300 \mathrm{~nm}$ & $350 \mathrm{~nm}$ \\
\hline 2 & 4.93 & 2.74 & 1.62 & 1.12 \\
2.5 & 3.28 & 1.82 & 1.08 & 0.74 \\
3 & 2.31 & 1.29 & 0.76 & 0.53 \\
3.5 & 1.71 & 0.95 & 0.56 & 0.39 \\
\hline
\end{tabular}

\section{Conclusions}

Deep-ultraviolet to mid-infrared polarizers based on $\mathrm{Al}$ nanowire metamaterials with hexagonally packed nanowire arrays have been reported. It is demonstrated that both the extinction ratio and insertion loss synchronously rise by increasing the diameter or decreasing the spacing of the $\mathrm{Al}$ nanowires, also, the extinction ratio and insertion loss simultaneously decline by decreasing the nanowire diameter or increasing the nanowire spacing. Furthermore, the mechanisms on the mode and the resonance characteristics of the Al nanowire metamaterials can be investigated in the near field regions by FDTD simulations. The electric field profile of the $\mathrm{Al}$ nanowire metamaterial exhibits a resonance behavior at the outer interface along the long axis of the nanowires and performs a standing wave mode for the spolarized light. In contrast, for the p-polarized light, very strong electric fields localized along the $\mathrm{x}$ direction can be observed, resulting from the electric field coupling between the adjacent nanowires. Importantly, for the periodical multilayer configuration of the $\mathrm{Al}$ nanowires, to synchronously achieve high extinction ratios and low insertion losses, we propose a $q$ factor (the ratio of the gap between two adjacent nanowires to the nanowire diameter) to determine the polarization characteristics of the optical polarizers, when the $q$ factor is fixed, it is found that the extinction ratio increases and the insertion loss decreases as the nanowire diameter decreases from $80 \mathrm{~nm}$ to $20 \mathrm{~nm}$, as a result, high extinction ratios and low insertion losses can be achieved synchronously. Moreover, the $q$ factor has a dominant influence on the polarization performance of the $\mathrm{Al}$ nanowire metamaterials, both the extinction ratio and the insertion loss decrease with increasing the $q$ factor at the same wavelengths for the same nanowire diameters. In this case, the value of $q$ factor should be chosen reasonably based on the expected performance tolerance of the optical polarizers, for longer wavelengths (e.g., in the mid-infrared spectral range), the polarizer presents very high extinction ratios and very low insertion losses for almost any $q$ factors, but for shorter wavelengths (in the visible and ultraviolet spectral regions), the $q$ should be a suitable value (e.g., 2 or 3 ) to achieve high extinction ratios and low insertion losses simultaneously. Additionally, consider the deep-ultraviolet spectral region, the cut-off wavelength for the polarizers presents obvious differences for the different substrate material. It is found that the cut-off wavelength remarkably decreases along short wavelengths with decreasing the refractive index of the material. Especially, the cut-off wavelength for the small nanowire diameter of $20 \mathrm{~nm}$ presents obvious blueshift from $310 \mathrm{~nm}$ to $198 \mathrm{~nm}$ when the refractive index decreases. In this case, the $\mathrm{Al}$ nanowire metamaterials embedded in the materials with lower refractive index exhibit high performance at the deepultraviolet wavelengths. Therefore, the high-performance polarizers operating down to the deep-ultraviolet wavelength range can be designed based on the optimal dimensions (e.g., $15 \mathrm{~nm}$ in diameter and the $q$ factor of 2 or 2.5) of $\mathrm{Al}$ nanowire metamaterials.

\section{Acknowledgements}

This work was supported by National Natural Science Foundation of China (51771186, 11574070, 11874012); The Fundamental Research Funds for the Central Universities (PA2018GDQT0006, JZ2017HGTB0187, JZ2017HGBZ0942); The Seventh Framework Program of the European Union for Research through Marie Curie Actions-International Incoming Fellowships (623473, 913473); Program 973 (2015GB103003); The Project of State Key Laboratory of Environment-friendly Energy Materials, Southwest University of Science and Technology (17FKSY0109); China Postdoctoral Science Foundation (2015M571918, 2017T100442); The European Union's Horizon 2020 research and innovation program under the Marie Skłodowska-Curie grant agreement (744817). 


\section{References}

[1] Xu M, Urbach H P, de Boer D K G and Cornelissen H J 2005 Opt. Express 132303

[2] Kim S H, Park J-D and Lee K-D 2006 Nanotechnology 174436

[3] Ge Z, Wu T X and Wu S-T 2008 Appl. Phys. Lett. 92051109

[4] Ge Z and Wu S-T 2008 Appl. Phys. Lett. 93121104

[5] Shin Y J, Wu Y-K, Lee K-T, Ok J G and Guo L J 2013 Adv. Opt. Mater. 1863

[6] Kim D 2005 Appl. Opt. 445398

[7] Wu Z, Powers P E, Sarangan A M and Zhan Q 2008 Opt. Lett. 331653

[8] Gruev V, Perkins R and York T 2010 Opt. Express 1819087

[9] Gardner E and Hansen D 2003 SID Symposium Digest Tech. 34 62

[10] Yu H, Oh Y, Kim S, Song S H and Kim D 2012 Opt. Lett. 37 3867

[11] Bird G R and Parrish M 1960 J. Opt. Soc. Am. 50886

[12] Wang J J, Chen L, Liu X, Sciortino P, Liu F, Walters F and Deng X 2006 Appl. Phys. Lett. 89141105

[13] Yamada I, Fukumi K, Nishii J and Saito M 2010 Opt. Lett. 35 3111

[14] Dai M, Wan W, Zhu X, Song B, Liu X, Lu M, Cui B and Chen Y 2015 Opt. Express 2315390

[15] Zografopoulos D C, Isic G, Kriezis E E and Beccherelli R 2016 J. Phys. D: Appl. Phys. 49195104

[16] Boehm S J, Kang L, Werner D H and Keating C D 2017 Adv. Funct. Mater. 271604703

[17] Kang W, Chu J, Zeng X and Fan Y 2018 Appl. Opt. 575230

[18] Ferraro A, Zografopoulos D C, Missori M, Peccianti M, Caputo R and Beccherelli R 2016 Opt. Lett. 412009

[19] Cetnar J S, Middendorf J R and Brown E R 2012 Appl. Phys. Lett. 100231912

[20] Imakita K, Kamada T, Fujii M, Aoki K, Mizuhata M and Hayashi S 2013 Opt. Lett. 385067

[21] Huang Z, Park H, Parrott E P J, Chan H P and PickwellMacPherson E 2013 IEEE Photonics Technol. L. 2581

[22] Huang Z, Parrott E P J, Park H, Chan H P and PickwellMacPherson E 2014 Opt. Lett. 39793

[23] Chen L, Wang J J, Walters F, Deng X, Buonanno M, Tai S and Liu X 2007 Appl. Phys. Lett. 90063111

[24] Kang J, Yun H-S, Jang H-I, Kim J, Park J H and Lee J-Y 2018 Adv. Opt. Mater. 61800205

[25] Kwon S, Lu D, Sun Z, Xiang J and Liu Z2016 Nanoscale 8 15850

[26] Alizadeh M H and Reinhard B M 2017 Opt. Express 2522897

[27] Asano K, Yokoyama S, Kemmochi A and Yatagai T 2014 Appl. Opt. $\mathbf{5 3} 2942$

[28] Siefke T, Kroker S, Pfeiffer K, Puffky O, Dietrich K, Franta D, Ohlidal I, Szeghalmi A, Kley E-B and Tuennermann A 2016 Adv. Opt. Mater. 41780

[29] Weber T, Kroker S, Kaesebier T, Kley E-B and Tuennermann A 2014 Appl. Opt. 538140

[30] Tropf W J and Thomas M E 1998 "Aluminum oxide $\left(\mathrm{Al}_{2} \mathrm{O}_{3}\right)$ revisited" in Handbook of optical constants of solids III, E. D. Palik, eds. (San Diego: Academic Press) p 662

[31] van de Hulst H C 1981 Light scattering by small particles (New York: Dover, 1981) p 67

[32] Smith D Y, Shiles E and Inokuti M 1998 "The optical properties of metallic aluminum", In Handbook of optical constants of solids, Palik, E. D. eds. (San Diego: Academic Press) p 396

[33] Saito M and Miyagi M 1989 J. Opt. Soc. Am. A 61895

[34] Kokhanovsky A A 2001 Optics of light scattering media: problems and solutions (Chichester: Springer) p 31 